\title{
Configurações
}

Revista de sociologia

14 | 2014

Para além da Governação. Políticas, práticas e discursos de inclusão e promoção da diversidade cultural

\section{¿Diversidad cultural o desigualdad social? Una aproximación crítica a la competencia cultural en la salud a partir de las necesidades sentidas por mujeres en contextos de diversidad, injusticia social y austeridad}

Diversidade cultural ou desigualdade social? Uma aproximação crítica à competência cultural na saúde a partir das necessidades sentidas por mulheres em contextos de diversidade, injustiça social e austeridade

Cultural diversity or social inequality? A critical approach to cultural competence in healthcare based on the felt needs of women in contexts of diversity, social injustice and austerity

Sonia Hernández Plaza

\section{OpenEdition}

Journals

Edición electrónica

URL: http://journals.openedition.org/configuracoes/2290

DOI: $10.4000 /$ configuracoes. 2290

ISSN: 2182-7419

Editor

Centro de Investigação em Ciências Sociais

Edición impresa

Paginación: 103-128

ISBN: 1646-5075

ISSN: 1646-5075

Referencia electrónica

Sonia Hernández Plaza, « ¿Diversidad cultural o desigualdad social? Una aproximación crítica a la competencia cultural en la salud a partir de las necesidades sentidas por mujeres en contextos de diversidad, injusticia social y austeridad », Configurações [En línea], 14 | 2014, Puesto en línea el 30 marzo 2015, consultado el 19 abril 2019. URL : http://journals.openedition.org/configuracoes/2290 DOI : 10.4000/configuracoes.2290

Este documento fue generado automáticamente el 19 abril 2019. 


\title{
¿Diversidad cultural o desigualdad social? Una aproximación crítica a la competencia cultural en la salud a partir de las necesidades sentidas por mujeres en contextos de diversidad, injusticia social y austeridad
}

\author{
Diversidade cultural ou desigualdade social? Uma aproximação crítica à \\ competência cultural na saúde a partir das necessidades sentidas por mulheres \\ em contextos de diversidade, injustiça social e austeridade \\ Cultural diversity or social inequality? A critical approach to cultural \\ competence in healthcare based on the felt needs of women in contexts of \\ diversity, social injustice and austerity
}

Sonia Hernández Plaza

En contextos de diversidad asociada a la inmigración, amplia evidencia empírica ha puesto de manifiesto que, en general, inmigrantes y minorías étnicas suelen tener peor salud, mayores difi cultades de acceso a los servicios de salud, y reciben cuidados de salud de peor calidad (Ingleby, Chiarenza, Devillé \& Kotsioni, 2012; Ingleby, Krasnik, Lorant \& Razum, 2012; Thomas \& Gideon, 2013; WHO Regional Offi ce for Europe, 2010). Un ejemplo particularmente grave de desigualdades en salud es el que afecta a mujeres embarazadas, madres, recién nacidos y niños, como grupos de particular vulnerabilidad. Investigaciones realizadas en diversos países europeos como Holanda (Zwart et al., 2010), Bélgica (Minsart, Englert \& Buekens, 2012), España (Luque, Bueno \& de Mateo, 2010; Luque, Gutiérrez \& Bueno, 2010) el Reino Unido (Lewis, 2007) y Portugal (Machado et al., 
2007), entre otros, han mostrado que las mujeres inmigrantes tienden a sufrir mayores tasas de mortalidad y morbilidad infantil y materna, y suelen mostrar peores indicadores de salud materno-infantil, tales como menor peso del bebé al nacer, mayor número de partos prematuros, mayores tasas de depresión postparto, o mayor frecuencia de complicaciones durante el embarazo y el parto, con severas implicaciones sobre la salud y el desarrollo infantil.

2 A la hora de explicar las desigualdades en salud en contextos de diversidad asociada a la inmigración, cabe destacar dos conjuntos de factores como determinantes fundamentales de dichas disparidades: 1) los procesos de exclusión social y las desigualdades socioeconómicas asociadas a la posición de desventaja social que habitualmente sufren los inmigrantes en los contextos receptores (Hernández-Plaza et al., 2010; Ingleby, 2012), considerados por la Comisión sobre los Determinantes Sociales de la Salud de la Organización Mundial de la Salud (OMS) como la principal causa de desigualdades en salud en poblaciones inmigrantes y minorías étnicas (CSDH, 2008); y 2) las desigualdades en el acceso y la calidad de los cuidados de salud (Ingleby, Chiarenza et al., 2012; WHO Regional Offi ce for Europe, 2010).

3 Una de las estrategias más conocidas para reducir las disparidades en salud y en el acceso y calidad de los cuidados de salud en inmigrantes y minorías étnicas es la promoción de la competencia cultural, entendida como "un conjunto congruente de comportamientos, actitudes y políticas que se manifiestan de manera conjunta en un sistema, agencia o entre profesionales, capacitando a dicho sistema, agencia o a aquellos profesionales para trabajar de manera efectiva en situaciones transculturales" (Cross et al., 1989: 13).

4 En el presente trabajo, tras delimitar conceptualmente la noción de competencia cultural y efectuar un breve recorrido histórico sobre el surgimiento y evolución de este concepto, se plantea una severa crítica a las políticas y prácticas de promoción de la competencia cultural dada su incapacidad para transformar las condiciones generadoras de desigualdad en el acceso a la salud en contextos de diversidad, particularmente en el actual contexto de crisis y austeridad.

5 La perspectiva crítica propuesta parte del análisis de un contexto específico, el Área Metropolitana de Lisboa, y se fundamenta en los resultados obtenidos en un proyecto de investigación ${ }^{1}$ desarrollado entre los años 2011 y 2013, periodo en el que Portugal es golpeado con particular crudeza por la crisis financiera y las medidas de austeridad impuestas por el gobierno de la nación y la denominada Troika, formada por el Fondo Monetario Internacional (FMI), el Banco Central Europeo (BCE) y la Comisión Europea (CE). Los principales objetivos de dicha investigación son: 1) evaluar las necesidades de los usuarios de cuidados de salud primarios en contextos de diversidad asociada a la inmigración en el Área Metropolitana de Lisboa, y 2) analizar críticamente la adecuación de la competencia cultural como estrategia para reducir las desigualdades en el acceso a la salud en dicho contexto, severamente golpeado por la crisis y las medidas de austeridad. Las necesidades de los usuarios, su naturaleza y determinantes, constituyen el referente fundamental a partir del cual se examina la relevancia y adecuación de las estrategias basadas en la promoción de la competencia cultural.

6 Los resultados detallados en el presente artículo corresponden específicamente a las necesidades percibidas o sentidas por las mujeres en el contexto objeto de estudio. Dada la escasa disponibilidad de datos acerca del impacto de la crisis y las medidas de austeridad sobre la salud y el acceso a la salud en Portugal, tal como ha sido 
recientemente advertido por el Observatorio Nacional de Sistemas de Salud (OPSS, 2014), los resultados que se presentan cubren una importante laguna en la literatura nacional e internacional, prestando especial atención a las necesidades sentidas por mujeres en contextos de diversidad asociada a la inmigración y condiciones de desventaja socioeconómica.

\section{Políticas y prácticas de inclusión de la diversidad cultural en el ámbito de la salud}

7 La creciente diversidad asociada a la globalización y las migraciones internacionales plantea un importante reto a las sociedades receptoras en lo que se refiere a la incorporación de los inmigrantes como ciudadanos en los diferentes ámbitos de la vida social, política, económica y cultural, incluyendo el acceso a los servicios públicos del Estado del Bienestar: la sanidad, la educación, los servicios sociales y las diversas prestaciones sociales (Bommes \& Geddes, 2000; Faist, 1994). En lo que se refiere específicamente al acceso a la salud, las políticas de incorporación de los inmigrantes se han fundamentado en una pluralidad de principios, entre los que cabe destacar la consideración de la salud y el acceso a la salud como derechos humanos fundamentales, la inclusión de la salud como parte esencial de las responsabilidades y derechos asociados a la ciudadanía, el concernimiento por la salud pública del conjunto de la población, o la preocupación por proporcionar servicios de salud culturalmente apropiados en contextos de multiculturalidad (Bommes \& Geddes, 2000; CE, 2006; Ingleby, Chiarenza et al., 2012; Padilla \& Pereira Miguel, 2009; Padilla, 2013; Padilla et al., 2013; Thomas \& Gideon, 2013; WHO Regional Office for Europe, 2010). El presente trabajo se centra específicamente en las prácticas fundamentadas en este último principio, revisando de manera crítica su pertinencia y adecuación en contextos de diversidad, crecientes desigualdades sociales y austeridad.

La preocupación por la provisión de servicios de salud culturalmente apropiados a inmigrantes y minorías étnicas surge hace más de cuatro décadas, entre finales de los años sesenta y comienzos de los setenta, principalmente asociada al fenómeno de la inmigración y la creciente diversidad cultural en países como Estados Unidos y Canadá (Sue et al., 2009). Desde entonces, han sido muy diversos los términos propuestos para hacer referencia a la necesidad de adaptar los servicios de salud a la diversidad cultural, pasando de nociones iniciales que ponían el acento en la "sensibilidad cultural" (cultural responsiveness, cultural sensitivity), al más reciente concepto de "competencia cultural" ( cultural competence).

\section{De la sensibilidad cultural a la competencia cultural}

9 Inicialmente, el término "sensibilidad cultural" (cultural sensitivity) ponía énfasis en la necesidad de conocer las características particulares de diferentes grupos culturales, incluyendo no solamente creencias y hábitos relacionados con la salud, sino también valores, normas y formas de interacción social, entre otros aspectos. Estos conocimientos eran frecuentemente recogidos en manuales que describían el modo en que los profesionales de la salud debían tratar a usuarios de grupos culturales específicos, como 
miembros de la cultura "A", "B" o "C" (Ingleby, 2011; WHO Regional office for Europe, 2010).

Sin embargo, muy pronto se pusieron de manifiesto las limitaciones de esta aproximación. Por un lado, dada la creciente diversidad cultural asociada al fenómeno migratorio global, resulta imposible disponer de manuales especializados sobre cada grupo cultural específico, siendo para los profesionales extremadamente complicado llegar a alcanzar un conocimiento profundo acerca de cada uno de los grupos a los que prestan atención en su práctica sanitaria (WHO Regional office for Europe, 2010).

11 Por otro lado, este enfoque inicial se sustenta en una noción esencializadora de cultura, duramente criticada desde la antropología, principalmente a partir de los trabajos de Geertz (1973) y Kleinman (Kleinman y Benson, 2006) entre otros, quienes cuestionan la existencia de "culturas" como entidades monolíticas, homogéneas, estáticas y claramente diferenciadas, enfatizando su carácter multidimensional, complejo, heterogéneo y dinámico, en continuo proceso de cambio y re-configuración. En consecuencia, este acercamiento inicial tiende a promover una imagen estereotipada y reduccionista de grupos sociales complejos, dando a entender que un determinado usuario, por el mero hecho de pertenecer a cierto grupo cultural, posee un conjunto de rasgos que caracterizan a todos los miembros de su grupo de manera unívoca, homogénea y estática.

ino "competencia cultural" (cultural competence) se propone en la década de los ochenta, en un intento por superar las limitaciones de las anteriores aproximaciones. Es precisamente a finales de los ochenta cuando se plantea una de las definiciones más conocidas y ampliamente aceptadas de "competencia cultural", entendida como "un conjunto congruente de comportamientos, actitudes y políticas que se manifiestan de manera conjunta en un sistema, agencia o entre profesionales, capacitando a dicho sistema, agencia o a aquellos profesionales para trabajar de manera efectiva en situaciones transculturales" (Cross et al., 1989: 13).

13 Tal como pone de manifiesto la conceptualización propuesta por Cross et al. (1989), y como han sugerido otros autores (Sue et al., 2009), es preciso diferenciar tres niveles de análisis y promoción de la competencia cultural: 1) nivel individual o interpersonal, de proveedor (profesionales de la salud) o tratamiento (p. ej. programa de prevención del VIH); 2) nivel organizacional, institucional o de agencia (centro de salud, hospital); y 3) nivel de sistema (sistema nacional de salud, sistema comunitario de cuidados de salud).

un primer momento, desde una perspectiva centrada en los profesionales, la mayor parte de las aproximaciones ponen énfasis en las cualidades que deben poseer los profesionales de la salud para proporcionar una atención culturalmente apropiada, centrándose principalmente en los conocimientos, habilidades y actitudes que deben tener o adquirir para trabajar de manera eficaz en situaciones transculturales (CampinhaBacote, 2001; Leininger, 1978; Suárez- Balcazar \& Rodakowski, 2007).

15 Un avance fundamental respecto a esta aproximación inicial viene dado por la constatación de la necesidad de implicar de manera global a las organizaciones proveedoras de servicios de salud, en su conjunto, pasando de un énfasis en los profesionales culturalmente competentes, a un mayor acento en la promoción de organizaciones de salud y servicios culturalmente apropiados. En este sentido, resulta particularmente relevante la propuesta planteada en el marco de los estándares CLAS ( Culturally and Linguistically Appropriate Services) en Estados Unidos (OMH, 2001), que proponen redefi nir la competencia cultural como "servicios cultural y lingüísticamente 
apropiados", caracterizados por "la capacidad de funcionar de manera efectiva como individuo y como organización, en el contexto de las creencias culturales, comportamientos y necesidades de los consumidores y sus comunidades", proporcionando "servicios de salud respetuosos y que respondan a sus necesidades culturales y lingüísticas" (OMH, 2001: 131).

Más allá de las organizaciones de salud y los servicios que prestan, algunas propuestas han subrayado la importancia de tomar en consideración las características del sistema de salud en su conjunto, tal como sugiere la OMS (WHO Regional Office for Europe, 2010), al poner el acento en la necesidad de desarrollar estrategias que permitan hacer frente a las desigualdades en salud vinculadas a las migraciones y la etnicidad desde el sistema de salud, considerado en su totalidad. De acuerdo con la OMS, un sistema de salud está formado por el conjunto de organizaciones, públicas y privadas, instituciones y recursos orientados a mejorar, mantener o restaurar la salud; incorporando servicios tanto personalizados como poblacionales, así como actividades dirigidas a influenciar las políticas y acciones de otros sectores a la hora de hacer frente a los determinantes sociales, ambientales y económicos de la salud. Desde esta aproximación, sería posible redefinir la competencia cultural como sistema de salud culturalmente apropiado.

Por otro lado, desde un enfoque integrador de las anteriores perspectivas, algunas conceptualizaciones proponen múltiples niveles de análisis y promoción de la competencia cultural, estrechamente interrelacionados entre sí, prestando atención tanto a las cualidades que deben poseer y/o adquirir los profesionales, como a los factores organizacionales implicados en la provisión de servicios culturalmente apropiados, como parte del sistema de salud, de manera más global (Cross et al., 1989; Sue et al., 2009; Weaver, 2008). La definición clásica de Cross y otros (1989), anteriormente referida, es un ejemplo paradigmático de esta aproximación integradora y multinivel.

\section{Sensibilidad a la diversidad, superdiversidad y equidad}

Más recientemente, el análisis crítico de las experiencias de promoción de la competencia cultural ha generado un profundo debate en torno a este concepto, siendo muy numerosos los autores que han planteado la necesidad de re-conceptualizarlo y desarrollar aproximaciones innovadoras y más comprehensivas (Balcazar et al., 2010; Chiarenza, 2012; García-Ramírez et al., 2012; Ingleby, 2011). En este sentido, perspectivas más recientes proponen redefinir el concepto de cultura, más allá de la etnicidad, incorporando en un sentido amplio todos los aspectos de la vida de una persona, es decir, su contexto en un sentido global incluyendo circunstancias presentes, situación socioeconómica, situación legal, entorno físico y social y vida cotidiana, en general (CE, 2006; WHO Regional Office for Europe, 2010). Así, por ejemplo, el Consejo de Europa en sus recomendaciones sobre servicios de salud en una sociedad multicultural (CE, 2006) adopta una definición amplia de cultura que reconoce la existencia de sub-categorías culturales basadas en dimensiones como el género, la educación, la ocupación, el status socioeconómico, la situación legal, o situaciones como la condición de sin hogar, o experiencias traumáticas comunes, entre otras.

19 En esta misma línea, algunos autores han sugerido la noción de sensibilidad a la diversidad (Ingleby, 2011; Renschler \& Cattacin, 2007), poniendo énfasis en la importancia de tomar en consideración el papel de múltiples dimensiones de la diversidad humana en la prestación de cuidados de salud, más allá de la etnicidad, incorporando también el 
género, la edad, la posición socioeconómica, la orientación sexual, la discapacidad, o el contexto comunitario, dadas sus implicaciones sobre las necesidades en el ámbito de la salud y el acceso a los servicios sanitarios. La creciente diversificación de la diversidad (Vertovec, 2007) asociada a las migraciones internacionales y la globalización ha dado lugar asimismo a recientes cuestionamientos acerca de la necesidad de repensar nuevos modelos de prestación de servicios de salud en la denominada era de la superdiversidad (Phillimore, 2011).

Por otro lado, diversas aproximaciones han propuesto un giro del énfasis en la diversidad cultural a un mayor acento en la reducción de las desigualdades y la promoción de la equidad en los servicios de salud (Hart, Hall \& Herwood, 2003; MFCCH, 2012). En este sentido, cabe destacar los recientes trabajos de la Task Force sobre Cuidados de Salud Culturalmente Competentes y Amigos de los Inmigrantes (MFCCH, 2012), desde la que se plantea un acercamiento alternativo a la competencia cultural a nivel individual y organizacional, que pretende trascender la noción de cultura vinculada a etnicidad, a través de la consideración de su intersección con dimensiones de género, clase, raza y edad, entre otras, poniendo énfasis en la noción de equidad tanto en el acceso como en la calidad de los cuidados de salud. Desde esta perspectiva se han propuesto una serie de estándares orientados a promover la equidad en las organizaciones de salud (hospitales, centros de salud) como principal estrategia para reducir las desigualdades en salud, y en el acceso y calidad de los servicios sanitarios, tomando en consideración las múltiples dimensiones de diversidad implicadas en dichas desigualdades.

\section{Desigualdades sociales y exclusión social}

21 El interés por la competencia cultural surge y se mantiene hasta nuestros días ante la creciente evidencia empírica que constata la existencia de importantes desigualdades en el estado de salud, el acceso y la calidad de los servicios de salud en poblaciones inmigrantes, en situación de clara asimetría respecto a la población autóctona (Ingleby, Chiarenza et al., 2012; Ingleby, Krasnik et al., 2012; Thomas \& Gideon, 2013; WHO Regional Offi ce for Europe, 2010). El objetivo último de las intervenciones culturalmente competentes es, por tanto, reducir las desigualdades en el acceso y la calidad de los servicios de salud, y en última instancia, contribuir a reducir las desigualdades en salud. No obstante, dichas desigualdades no se relacionan exclusivamente con factores culturales. De acuerdo con la OMS, en base al trabajo desarrollado por la "Comisión sobre los Determinantes Sociales de la Salud" (CSDH, 2008), la principal causa de desigualdades en la salud y el acceso a los cuidados de salud en inmigrantes y minorías étnicas son los procesos de exclusión social.

22 En este sentido, resulta particularmente esclarecedora la definición que ofrece la Red de Conocimiento sobre Exclusión Social (SEKN: Social Exclusion Knowledge Network) de la Comisión sobre los Determinantes Sociales de la Salud de la OMS, según la cual: "la exclusión consiste en una serie de procesos dinámicos y multidimensionales, estrechamente relacionados con las relaciones desiguales de poder que interactúan en torno a cuatro dimensiones principales - económica, política, social y cultural- y a diferentes niveles, incluyendo los niveles individual, familiar, comunitario, nacional y global. El resultado de dichos procesos es un continuo de inclusión/exclusión caracterizado por un acceso desigual a los recursos, capacidades y derechos que conduce a las desigualdades en la salud (SEKN, 2008: 2). 

entre los años 2011 y 2014 se llevan a cabo drásticos recortes en los presupuestos dedicados a sanidad pública ${ }^{3}$, implementándose duras medidas de austeridad entre las que se encuentra el incremento de las tasas moderadoras que los usuarios han de pagar por la utilización del Servicio Nacional de Salud (SNS) ${ }^{4}$. De acuerdo con las exigencias de la Troika, en el año 2012 se incrementa drásticamente el coste directo de la atención sanitaria para los usuarios del sistema público de salud, llegándose a duplicar el valor de las tasas moderadoras en algunos servicios, tal como sucede en las consultas de atención primaria, puerta de entrada en el SNS, que pasan de 2,25€ en 2011 a $5 €$ en 2012 hasta la actualidad; o en las consultas de urgencia en hospitales, que pasan de 9,6€ en 2011 a $20 €$ en $2012,20,6 €$ en 2013 y $20,65 €$ en $2014^{5}$. produce en un contexto caracterizado por el progresivo y sustancial aumento de desempleo, que prácticamente se ha duplicado durante los años de la crisis, pasando del $8,6 \%$ en 2006, al 16,4\% en 2013, según datos del EUROSTAT; y por un paralelo incremento 
de la población en riesgo de pobreza y exclusión social, que ha ascendido de un $25 \%$ en 2006 al 27,4\% en 2013 en la población general, y de un 25,5\% en 2006 a un estremecedor $31,6 \%$ en 2013 en lo que se refiere específicamente a infancia en riesgo de pobreza y exclusión social, de nuevo de acuerdo con el EUROSTAT. la revisión de las categorías de exención de pago, regulada mediante el Decreto Ley n.ำ 113/2011 y el Decreto Ley nº 128/2012, según los cuales tienen derecho a la exención por insuficiencia económica, a efectos de exención de pago de tasas moderadoras y otros pagos derivados del acceso a los servicios de salud, los usuarios que integren un agregado familiar cuyos ingresos medios mensuales, dividido por el número total de personas a quien cabe la dirección del agregado familiar, sea igual o inferior a 1,5 veces el valor del Índice de Apoyos Sociales (IAS), correspondiente en 2014 a 628,83€. Como veremos más adelante, los resultados obtenidos en nuestro trabajo permiten cuestionar la capacidad de dichas exenciones para garantizar el acceso universal a la salud, en el actual contexto de crisis. desaparición de la fi gura del mediador intercultural en centros de salud y hospitales, que había sido identificada como buena práctica en salud y migraciones en Portugal (Da Silva y Martingo, 2007; Padilla et al., 2009). En el presente, la figura del mediador intercultural únicamente existe en el ámbito de los servicios sociales comunitarios en algunos municipios del Área Metropolitana de Lisboa, como parte del "Proyecto de mediación intercultural en servicios públicos" (MISP), promovido por el Alto Comisariado para la Inmigración y el Diálogo Intercultural (ACIDI), actualmente Alto Comisariado para las Migraciones (ACM), en colaboración con diversas Cámaras Municipales y Asociaciones de Inmigrantes.

\section{Investigación-acción participativa: notas metodológicas}

Los resultados que se presentan y analizan en este apartado son fruto de un proyecto de investigación-acción participativa basada en la comunidad (García- Ramírez et al., 2012; Montero, 2006) ${ }^{6}$, a través de una coalición comunitaria formada por un equipo interdisciplinar de investigadoras sociales de diversas disciplinas (psicología comunitaria, sociología, ciencia política, antropología), implicado en un conjunto de proyectos sobre salud y migraciones ${ }^{7}$ y diversos actores en el ámbito de la salud en contextos de diversidad asociada a la inmigración, principalmente: profesionales de la salud, trabajadores sociales, mediadores interculturales, asociaciones comunitarias y organizaciones no gubernamentales (ONG).

31 El contexto objeto de estudio es el Área Metropolitana de Lisboa, donde reside el 53,4\% de la población extranjera en Portugal (SEF, 2012); siendo asimismo la región del país con mayores tasas de desempleo, situado en el 18,5\% de la población en 2013 según datos del EUROSTAT. Dentro de este contexto amplio, la investigación se centra principalmente en los municipios de Amadora y Sintra, caracterizados por una concentración particularmente elevada de inmigrantes y sus descendientes, así como por altos niveles de vulnerabilidad y desventaja socio-económica. En Amadora, gran parte de la población inmigrante, principalmente la procedente de los $\mathrm{PALOP}^{8}$, se concentra en barrios 
segregados ubicados dentro del núcleo urbano o en el extrarradio, donde predominan las viviendas construidas por los propios moradores, comúnmente denominadas "barracas"; mientras que en Sintra, los inmigrantes tienden a vivir dispersos, con frecuencia en viviendas compartidas por varias familias para poder hacer frente a los costes del alquiler.

La investigación recurre a una aproximación multi-método (Creswell, 2009; Tashakkori y Teddlie, 2003), combinando técnicas de recogida de información tanto cualitativa como cuantitativa. La primera fase, de naturaleza cualitativa, consistió en la realización de un total de 52 entrevistas en profundidad: 13 entrevistas a informantes clave en el ámbito de la salud y la inmigración; 32 entrevistas a usuarios, tanto autóctonos como inmigrantes de los principales países de origen en los contextos objeto de estudio ( 6 caboverdianos, 7 de otros PALOP, 6 brasileiros y 13 autóctonos, 7 de ellos con padres africanos), 16 en el municipio de Amadora, y 16 en el municipio de Sintra; y 7 entrevistas a profesionales de la salud, 4 médicos y 3 enfermeros, 4 en el municipio de Amadora y 3 en el municipio de Sintra, incluyendo tanto profesionales autóctonos como extranjeros.

La segunda fase de la investigación, de naturaleza cuantitativa, consistió en la realización de una encuesta a una muestra formada por 253 usuarios de cuidados de salud primarios. Dada la particular vulnerabilidad de la salud materno-infantil en el actual contexto de crisis (Hernández-Plaza et al., 2014; Padilla et al., 2013), el análisis que se presenta a continuación presta especial atención a las necesidades de las mujeres en edad reproductiva como usuarias de cuidados de salud primarios. Del total de la muestra, 125 son mujeres en edad reproductiva ${ }^{9}$, de entre 18 y 49 años; de las cuales 70 residen en Amadora, 43 en Sintra, 10 en Lisboa y 2 en Cascais. En cuanto al país de nacimiento, 24 son de Brasil, 24 de Cabo Verde, 32 de otros PALOP (Angola, São Tomé y Guinea Bissau), y 45 son mujeres nacidas en Portugal, de las cuales 22 son de origen africano. La mayoría de las entrevistadas tienen hijos (69,6\%), predominando las mujeres jóvenes (de 18 a 28 años). Una descripción más detallada del perfil socio-demográfico de las entrevistadas se presenta en la tabla 1.

Tabla

Perfil socio-demográfico de la muestra de mujeres $(n=125)$ 


\begin{tabular}{|l|c|c|}
\hline VARIABLES SOCIO-DEMOGRÁFICAS & \% & N \\
\hline País de nacimiento & & \\
\hline Portugal, autóctono & 18,4 & 23 \\
Portugal, descendiente & 17,6 & 22 \\
Cabo Verde & 19,2 & 24 \\
Otros PALOP & 25,6 & 32 \\
Brasil & 19,2 & 24 \\
\hline Municipio (Área Metropolitana de Lisboa) & & \\
\hline Amadora & 56 & 70 \\
\hline Sintra & 34,4 & 43 \\
Otro & 9,6 & 12 \\
\hline Edad & & \\
\hline 18-30 años & 50,4 & 63 \\
31-40 años & 30,4 & 38 \\
\hline $41-49$ años & 19,2 & 24 \\
\hline
\end{tabular}

\begin{tabular}{|l|c|c|}
\hline VARIABLES SOCIO-DEMOGRÁFICAS & \% & N \\
\hline Nivel educativo & & \\
\hline Sin estudios & 4,1 & 5 \\
\hline Estudios primarios & 48,7 & 59 \\
\hline Enseñanza secundaria & 26,4 & 32 \\
\hline Formación profesional & 1,7 & 2 \\
\hline Estudios universitarios (no concluidos) & 3,3 & 4 \\
\hline Estudios universitarios (concluidos) & 6,6 & 8 \\
\hline Estudios de posgrado & 9,1 & 11 \\
\hline Situación laboral & & \\
\hline Empleado & 41,5 & 51 \\
\hline Desempleado & 47,2 & 58 \\
\hline Estudiante & 8,9 & 11 \\
\hline Incapacidad laboral & 0,8 & 1 \\
\hline Trabajo doméstico (no remunerado) & 1,6 & 2 \\
\hline & & $\mathrm{N}=125$ \\
\hline VARIABLES MIGRATORIAS & & \\
\hline Años en Portugal & & \\
\hline < 5 años & 30,1 & 22 \\
\hline $5-10$ años & 35,6 & 26 \\
\hline$>10$ años & 34,2 & 25 \\
\hline Situación legal & & \\
\hline Permiso de residencia & 62,8 & 54 \\
\hline Nacionalidad portuguesa & 20,9 & 18 \\
\hline Visado de estudiante & 8,1 & 7 \\
\hline En trámite & 4,7 & 4 \\
\hline Indocumentado & 3,5 & 3 \\
\hline & & \\
\hline
\end{tabular}

Los resultados que se analizan a continuación corresponden específicamente a la evaluación de las necesidades sentidas o percibidas ${ }^{10}$, mediante dos ítems en los cuales los entrevistados debían indicar las tres dificultades más importantes experimentadas en el acceso a los cuidados de salud primarios (experiencia con su centro de salud) y en la relación con los profesionales de la salud. Las opciones de respuesta se elaboraron con base en la información cualitativa obtenida en la primera fase de la investigación, 
incluyendo necesidades y dificultades a múltiples niveles (personal, relacional, organizacional, comunitario, socioeconómico, sistema de salud, nivel cultural / proceso migratorio) ${ }^{11}$, incluyendo cuestiones relacionadas con la falta de sensibilidad cultural, sensibilidad hacia la inmigración, sensibilidad hacia la situación socioeconómica del usuario, así como la discriminación racial, por ser inmigrante, por vivir en barrios marginalizados, por la lengua o por razones económicas. Se incorporó además un ítem abierto que permitía indicar cualquier otra necesidad o dificultad sentida por los entrevistados, no contemplada previamente entre las opciones de respuesta. Asimismo, se analizan indicadores de las tres necesidades prioritarias identificadas, a partir de tres ítems del cuestionario, formado por un total de 117 ítems. La recogida de datos se efectuó entre los meses de abril y junio del 2013, durante la implementación de las medidas de austeridad impuestas por la Troika ${ }^{12}$.

\section{Evaluación de necesidades en contextos de diversidad}

La evaluación de las necesidades percibidas por las mujeres, como usuarias de cuidados de salud primarios, revela que las tres necesidades identificadas como prioritarias por las participantes en el estudio ${ }^{13}$ son: 1 ) Dificultades para tener una consulta médica cuando se precisa, por las largas listas de espera $(33,1 \%) ; 2$ ) No tener médico de familia $(32,5 \%)$; y 3 ) Problemas de acceso a los medicamentos, por no poder pagar su coste $(32,3 \%)$.

A continuación, más de un $20 \%$ de las entrevistadas destacan las siguientes necesidades: 4) Dificultades a la hora de pedir cita médica $(31,5 \%)$; 5) Falta de continuidad en la relación con los médicos $(29,2 \%) ; 6)$ Elevado tiempo de espera en el centro de salud $(26,6 \%$ ); 7) Dificultades para pagar los exámenes complementarios y test diagnósticos (21\%); y 8) Dificultades para pagar las consultas médicas $(20,2 \%)$.

Tal como puede observarse en la tabla 2, los problemas que menos preocupan a las participantes en el estudio son: 1) la falta de sensibilidad cultural de los profesionales de la salud $(0,8 \%)$ y 2$)$ la discriminación por su situación económica $(0,8 \%)$. Ninguna de las mujeres encuestadas señala como problema la falta de sensibilidad hacia la inmigración, la discriminación por la lengua, o la discriminación, de un modo general.

TABLA 2.

EVALUACIÓN DE NECESIDADES PERCEBIDAS 

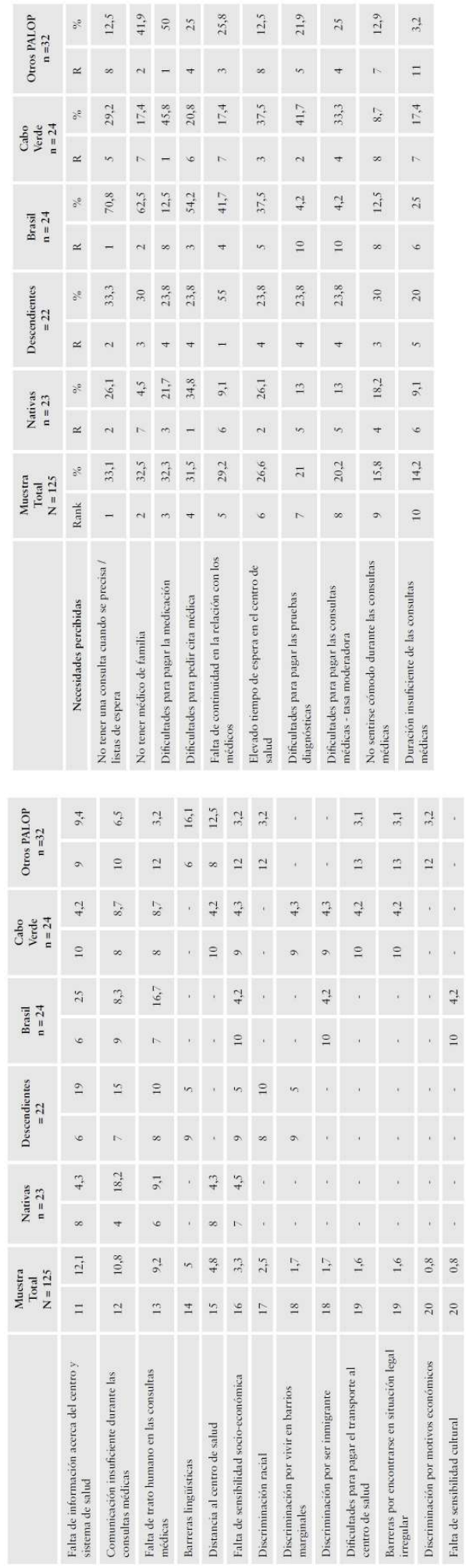


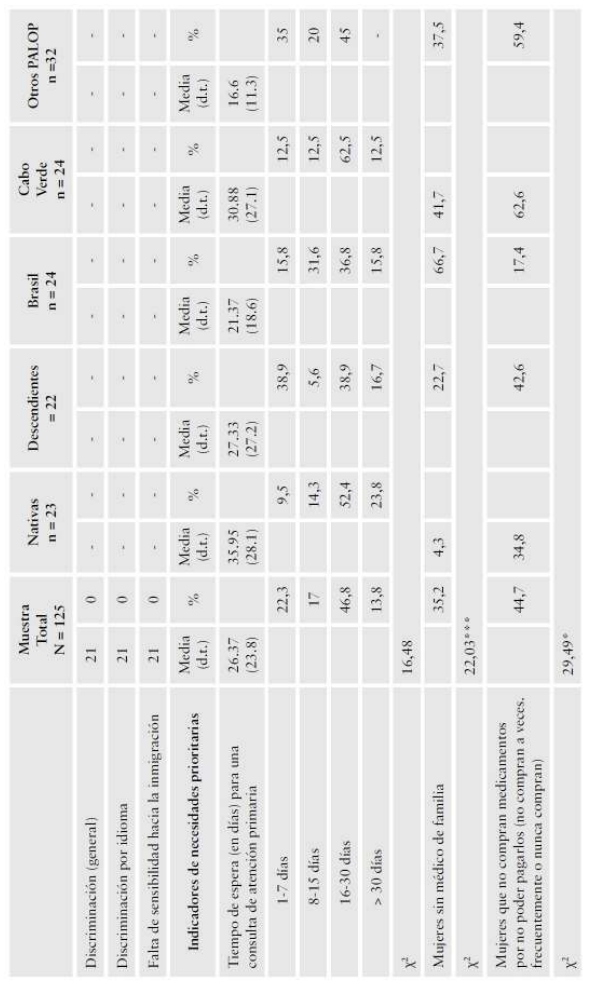

Respecto a los indicadores de las tres necesidades prioritarias identificadas, tal como puede observarse en la tabla 2, las entrevistadas refieren una espera media de más de 26 días ( $M=26.3$, D.T.=23.8) para ser atendidas en una consulta de atención primaria; el 35,2\% no tienen médico de familia; y el $44,7 \%$ no pueden comprar los medicamentos prescritos por los médicos (a veces, frecuentemente o nunca compran) debido a su situación de precariedad económica. En la tabla 3 se recogen algunas narrativas que ilustran las tres necesidades prioritarias identificadas, correspondientes a la fase cualitativa del proyecto de investigación.

TABLA 3.

NECESIDADES SENTIDAS COMO PRIORITARIAS: NARRATIVAS 
Problemas de acceso a las consultas: listas de espera en cuidados de salud primarios - "Las consultas pueden demorar un mes o más" (Mujer portuguesa, madre de cuatro niños, desempleada, Santa Filomena, Amadora)

- "Fui el viernes al médico y tengo consulta para dentro de un mes" (Mujer descendiente, padres caboverdianos, estudiante, Sintra)

- "Cuando tenemos algún problema de salud, llamo a Salud 24 horas, o voy directamente al hospital. Raramente voy a las consultas del centro de salud porque siempre tengo muchas dificultades para conseguir cita" (Mujer portuguesa, madre de dos niños, Sintra)

Problemas de acceso a las consultas: listas de espera en cuidados de salud primarios - "Las consultas pueden demorar un mes o más" (Mujer portuguesa, madre de cuatro niños, desempleada, Santa Filomena, Amadora)

- "Fui el viernes al médico y tengo consulta para dentro de un mes" (Mujer descendiente, padres caboverdianos, estudiante, Sintra)

- "Cuando tenemos algún problema de salud, llamo a Salud 24 horas, o voy directamente al hospital. Raramente voy a las consultas del centro de salud porque siempre tengo muchas dificultades para conseguir cita" (Mujer portuguesa, madre de dos niños, Sintra)

Problemas de acceso al médico de familia

- "Muchos no tienen médico de familia (...) hay mucha población para los médicos. Creo que esto es la principal barrera (...) no hay médicos para atender tanta población, y por eso las consultas están tan lejanas, por eso hay personas que no tienen médicos, que no pueden hacer una consulta hace mucho tiempo... Porque no hay médicos, sencillamente esa es la respuesta" (Médico, Amadora)

- "Aquí no tenemos médico de familia. Aquí, vamos a la consulta... y un día hay un médico, otro día hay otro médico, y otro día otro, jes muy molesto!” (Mujer caboverdiana, nacionalidad portuguesa, desempleada, Cova da Moura, Amadora)

- "No tengo médico de familia así que tengo que esperar, donde me puedan encajar" (Mujer brasileña, madre de un bebé, autorización de residencia, desempleada, Amadora)

\section{Dificultades para pagar la medicación}

- "Hay mucha gente que, porque no les alcanza el dinero, tienen que disminuir muchos medicamentos. Muchos no toman los medicamentos, y de hecho muchos dejaron de venir a consulta precisamente por eso (...) por su situación económica, no tienen dinero para hacerse exámenes, no tienen dinero para comprar los medicamentos no tienen dinero para comer" (Médico, Amadora)

- “Tengo diabetes desde hace cuatro años. La hipertensión empezó ahora, y el colesterol hace un año o dos (...) Siempre tengo dificultades para comprar medicación (...) A veces estoy dos o tres meses sin comprar los medicamentos para el colesterol, y no puedo estar sin ellos (...) Cuando no tengo dinero no compro medicamentos, cuando tengo dinero, los compro" (Mujer de São Tomé y Príncipe, autorización de residencia permanente, desempleada, barrio 6 de Maio, Amadora)

- "Todavía no he comprado una caja de medicinas que costaba 13 ” porque aún no he cobrado la pensión (...) compro lo menos posible (...) Si comprara muchos medicamentos, ¿entonces qué hago? ¡no llega! (Mujer portuguesa, jubilada, Sintra)

- "Tengo recetas en casa, pero no tengo condiciones para comprarlas" (Mujer caboverdiana, enferma crónica con hipertensión, indocumentada, desempleada, Santa Filomena, Amadora)

Las comparaciones en función del país de origen, orientadas a identificar posibles desigualdades, revelan la existencia de diferencias estadísticamente significativas en el acceso al médico de familia y la medicación. Por un lado, las mujeres brasileiras son el grupo con menor acceso al médico de familia de todos los analizados, concretamente el $66,7 \%$ de las entrevistadas de este origen no tienen médico de familia. A continuación se encuentran las mujeres caboverdianas y procedentes de otros PALOP, con porcentajes de $41,7 \%$ y $37,5 \%$, respectivamente, sin médico de familia. De manera muy significativa, las menos afectadas por esta problemática son las mujeres autóctonas, de las cuales solamente un 4,3\% carece de médico de familia (es decir, un 95,7\% de las mujeres 
autóctonas entrevistadas tiene médico de familia). Dado que casi el 70\% de las mujeres inmigrantes entrevistadas llevan más de 5 años en Portugal, no es posible atribuir estas diferencias al tiempo de residencia, ya que se trata en su mayoría de mujeres con asentamiento de media-larga duración en el país.

Por otro lado, son las mujeres caboverdianas y procedentes de otros PALOP las que sufren en mayor medida problemas de acceso a la medicación que precisan por carecer de recursos económicos suficientes. Las mujeres autóctonas y descendientes también se ven seriamente afectadas por estas dificultades; reportadas en menor medida por las inmigrantes brasileiras, probablemente debido a que la mayor parte de las mujeres de esta procedencia se encontraban en situación de empleo en el momento de realización de la entrevista.

41 Diferencias similares se observan en las necesidades percibidas como prioritarias por cada grupo, en función del país de origen, destacando de manera particular la escasa preocupación de las mujeres autóctonas por no tener médico de familia, problemática que como hemos visto afecta a un porcentaje reducido de este grupo; así como la mayor preocupación de las mujeres caboverdianas y de otros PALOP por cuestiones relacionadas con las dificultades para pagar los medicamentos, consultas médicas y pruebas diagnósticas que precisan, debido a su grave situación de precariedad socioeconómica.

En lo que se refiere a los problemas menos relevantes para las mujeres entrevistadas, existe total acuerdo, con independencia de su origen, destacando claramente la menor preocupación por: 1) la falta de sensibilidad hacia la inmigración; 2) la falta de sensibilidad cultural de los profesionales de la salud; 3) y la discriminación por razones económicas, por ser inmigrante, por la lengua o por vivir en un barrio social. Estas cuestiones no son preocupaciones prioritarias para las participantes en el estudio, con independencia de su país de origen.

\section{¿Diversidad cultural o desigualdad social?}

43 Las necesidades de las mujeres usuarias de cuidados de salud primarios en los contextos objeto de estudio se articulan en torno a dos dimensiones centrales. En primer lugar, las desigualdades sociales y la desventaja socioeconómica, asociadas a las dificultades de acceso a los medicamentos, consultas médicas y pruebas diagnósticas por no poder pagar su coste, en el actual contexto de crisis y austeridad, aumento del desempleo y la pobreza, e incremento del coste directo de los servicios públicos de salud para los usuarios, impuesto por la Troika.

44 Aunque se mantiene la exención por insuficiencia económica para personas y familias con reducidos ingresos, las necesidades percibidas identificadas en nuestro estudio ponen de manifiesto la incapacidad de dichas exenciones para garantizar el acceso a la salud a todas las personas que viven en condiciones de precariedad económica. En esta misma línea, informes recientes del Observatorio Portugués de Sistemas de Salud (OPSS, 2014) han subrayado la necesidad de mejorar la accesibilidad del SNS en el actual contexto de crisis, en lo que se refiere específicamente al coste de las tasas moderadoras relativas a consultas médicas, urgencias y pago de medicamentos (OPSS, 2014).

En segundo lugar, y en estrecha relación con la dimensión anterior, las necesidades identificadas como prioritarias se relacionan con severas deficiencias del Servicio Nacional de Salud, en lo que se refiere a listas y tiempos de espera, así como a la 
incapacidad de asegurar el derecho de acceso al médico de familia de manera universal, en un contexto caracterizado por los drásticos recortes en sanidad pública, bajo los dictados de la Troika. Si bien el SNS presentaba ya con anterioridad importantes carencias y deficiencias, la disminución de recursos humanos sanitarios, debido a la reducción de las contrataciones y a la salida de profesionales para trabajar en el sistema privado por el deterioro continuo de las condiciones laborales y salariales, está contribuyendo a agravar dichas deficiencias, reduciendo la calidad y adecuación de la atención sanitaria prestada, y acentuando la insuficiencia estructural de recursos humanos sanitarios como problema recurrente en el SNS en Portugal (Masanet et al., 2014).

46 Aunque la crisis, las medidas de austeridad y el deterioro de las condiciones socioeconómicas asociado al aumento del desempleo y la pobreza están golpeando con crudeza tanto a autóctonos como a inmigrantes, los resultados de nuestro estudio muestran que son estos últimos quienes están sufriendo mayores barreras económicas de acceso al sistema público de salud, asociadas a una mayor precariedad socioeconómica y a condiciones más extremas de pobreza y exclusión social.

47 Asimismo, se observa una clara desigualdad en el acceso al médico de familia, significativamente menor en el caso de las usuarias inmigrantes, que por tanto experimentan en mayor medida una falta de continuidad en la relación con los profesionales de la salud, que dificulta gravemente la posibilidad de establecer una relación de confianza, limitando las oportunidades de los profesionales de la salud para conocer las condiciones personales, familiares, económicas, sociales, laborales y culturales de las mujeres inmigrantes, como dimensión absolutamente esencial en el ámbito de la salud materno-infantil, imprescindible para garantizar una adecuada calidad de los cuidados de salud a mujeres, embarazadas, madres, bebés e infancia en contextos de diversidad.

Esta desigualdad en el acceso al médico de familia podría ser considerada una forma de discriminación institucionalizada, ejercida por el propio sistema de salud, que tendería a conceder un tratamiento preferencial, ofreciendo cuidados de salud de mayor calidad, a los usuarios autóctonos. Asimismo, supone una clara violación, en la práctica, del derecho reconocido en Portugal por el Despacho n.. 25.360/2001, que establece las condiciones de acceso al SNS por parte de los inmigrantes, otorgando a todos los extranjeros que residen de manera legal en este país el derecho de acceso al SNS, en condiciones de igualdad de tratamiento. Los resultados obtenidos en nuestro estudio muestran el claro incumplimiento en la práctica de dicho derecho, poniendo de manifiesto la existencia de importantes desigualdades en el acceso al SNS, en detrimento de los usuarios inmigrantes.

49 Aunque los resultados analizados en este artículo corresponden a una reducida muestra de mujeres, con el consiguiente sesgo de género y limitaciones a la hora de generalizar resultados, las necesidades identificadas son consistentes con las detectadas en los proyectos más amplios, de naturaleza multimétodo, en los que se inserta el presente trabajo, tanto en el ámbito de la salud materno-infantil (Hernández-Plaza et al., 2014; Masanet et al., 2014; Padilla et al., 2013), como en el área más general de los cuidados de salud primarios (Hernández-Plaza, 2014); resultando consistentes asimismo con otros trabajos e informes recientes que llaman la atención sobre los efectos negativos de la crisis y las medidas de austeridad sobre la salud y el SNS (Figueiredo, 2012, 2014; ONDR, 2013; OPSS, 2014), que si bien presentaba ya serias deficiencias antes de las medidas de 
austeridad, está viendo gravemente constreñida su capacidad de respuesta en tiempos de creciente necesidad.

\section{Conclusiones}

Para concluir este artículo, retomamos la pregunta planteada páginas atrás, en la que se cuestionaba: en el actual contexto de crisis y austeridad, ¿en qué medida pueden contribuir las políticas y prácticas de promoción de la competencia cultural a la reducción de las desigualdades en salud en contextos de diversidad asociada a la inmigración? En el caso particular del Área Metropolitana de Lisboa, los resultados del estudio que se ha presentado hacen necesario plantear una severa crítica a este tipo de políticas y prácticas, dada su incapacidad para transformar las condiciones generadoras de desigualdad en el acceso a la salud y los servicios sanitarios, más relacionadas con las desigualdades sociales y económicas, la estratificación social, las asimetrías de poder, las deficiencias del sistema público de salud, la posible discriminación institucionalizada en el acceso al médico de familia, y las políticas de austeridad y desmantelamiento del Estado del Bienestar (IOE, 2014; Navarro, 2014a, 2014b, 2012), que con la diversidad cultural.

51 Cabe recordar la palabras del acreditado Colectivo IOE, formado por Walter Actis, Carlos Pereda y Miguel Ángel de Prada, al destacar que "existe el riesgo de "culturizar" excesivamente la cuestión de los grupos étnicos, despojando a la problemática de sus alcances económicos y políticos (...) La relación entre grupos culturales está mediada por la distinta posición social de los mismos, por tanto, la interculturalidad no puede entenderse al margen de los fenómenos de poder y división de clases" (IOE, 1998: 243).

La gobernanza de la diversidad cultural en salud ha de ir por tanto inexorablemente unida a la gobernanza de las desigualdades sociales y económicas, y a la necesaria lucha contra la pobreza y la exclusión social, a menudo sufridas con mayor severidad por los inmigrantes; reconociendo las desigualdades en la distribución del poder y los recursos como característica estructural del capitalismo (IOE, 2014), y tomando en consideración el papel de las políticas sociales y económicas neoliberales como determinante del escandaloso crecimiento de las desigualdades en el actual contexto de crisis y desmantelamiento del Estado del Bienestar (Navarro, 2014a, 2012).

\section{Agradecimientos}

53 La autora agradece a todas las mujeres, asociaciones comunitarias, ONGs, profesionales de la salud, mediadores interculturales, trabajadores sociales e investigadores que han contribuido al desarrollo del estudio presentado en el presente artículo, en tiempos de crisis y extrema dificultad en Portugal. 


\section{BIBLIOGRAFÍA}

BALCAZAR, Fabricio; SUAREZ-BALCAZAR, Yolanda; WILLIS, Christopher \& ALVARADO, Francisco (2010), “Cultural competence: A review of conceptual frameworks", En Fabricio Balcazar, Yolanda Suarez-Balcazar, Tina Taylor-Ritzler, \& Christopher Keys (Eds.), Race, culture, and disability: Rehabilitation science and practice, Boston, Jones \& Bartlett: 281-305.

BOMMES, Michele \& GEDDES, A. (2013), Immigration and Welfare: Challenging the borders of the Welfare State, London, Routledge.

BRADSHAW, Jonathan (1972), “The concept of social need”. New Society, 30: 640-643.

CAMPINHA-BACOTE, Josepha (2001), "A model of practice to address cultural competence in rehabilitation nursing”, Rehabilitation Nursing, 26, 1: 8-11.

CE (2006), Recommendation Rec2006(18) of the Committee of Ministers to Member States on health services in a multicultural society, Strasbourg, Council of Europe.

CHIARENZA, Antonio (2012), "Developments in the concept of cultural competence", En David Ingleby, Antonio Chiarenza, Walter Devillé \& Ioanna Kotsioni (Eds), Inequalities in health care for migrants and ethnic minorities , Antwerp, Belgium: 66-81.

CRESWELL, John (2009), Research design. Qualitative, quantitative and mixed methods approaches, Third Edition, Thousand Oaks, Sage.

CROSS, Terry; BAZRON, Barbara; DENNIS, Karl \& ISAAC, Mareasa (1989), Towards a culturally competent system of care: a monograph on effective services for minority children who are severely emotionally disturbed, Volume I, Washington, DC: CASSP.

CSDH (2008), Closing the gap in a generation: health equity through action on the social determinants of health. Final report of the Commission on the Social Determinants of Health, Geneva, World Health Organization.

DA SILVA, António Carlos \& MARTINGO, Carla (2007), “Unidades de saúde amigas dos imigrantes: uma resposta ao desafio da multiculturalidade em Portugal”, Migrações, 1: 155-159.

FAIST, Thomas (1994), "Immigration, integration and the ethnicization of politics", European Journal of Political Research, 25: 439-459.

FIGUEIREDO, Gonçalo (2014), "Mental health in Portugal in times of austerity”, The Lancet Psychiatry, 1, 2: 109-110.

FIGUEIREDO, Gonçalo (2012), “Cuts in Portugal's NHS could compromise care”, The Lancet, 379, 400.

GARCÍA RAMÍREZ, Manuel; HERNÁNDEZ PLAZA, Sonia; ALBAR, María Jesús; LUQUERIBELLES, Violeta \& SUÁREZ-BALCÁZAR, Yolanda (2012), "Building healthcare stakeholder coalitions: A community psychology approach to user involvement for migrant populations", En David Ingleby, Antonio Chiarenza, Walter Devillé and Ioanna Kotsioni (Eds.), Inequalities in health care for migrants and ethnic Minorities, COST Series on Health and Diversity, Volume II: 188-204, Antwerp/ Apeldoorn, Garant.

GEERTZ, Clifford (1973), The interpretation of cultures, New York, Basic Books. 
HART, Angie; HALL, Valerie \& HERWOOD, Flis (2003), "Helping health and social care professions to develop an "inequalities imagination". A model for use in education and practice", Journal of Abnormal Nursing, 41, 5: 480-489.

HERNÁNDEZ-PLAZA, Sonia (2014), Final Report Summary: PSYSPOCUC “Meeting the healthcare needs of culturally diverse populations: A psycho-sociopolitical approach to cultural competence in health professionals". Marie Curie Actions FP7-PEOPLE-2010-IEF (Ref. 272976), 7th Research Framework Programme, European Commission, European Union.

HERNÁNDEZ-PLAZA, Sonia; PADILLA, Beatriz; ORTIZ, Alejandra \& RODRIGUES, Elsa (2014), “The value of grounded theory for disentangling inequalities in maternal-child healthcare in contexts of diversity: a psycho-sociopolitical approach", Psychosocial Intervention, 23: 125-133.

HERNÁNDEZ-PLAZA, Sonia; GARCÍA-RAMÍREZ, Manuel; CAMACHO, Carlos, \& PALOMA, Virginia (2010), "New settlement and wellbeing in oppressive contexts: a liberation psychology approach", En Stuart Carr (Ed.), The psychology of global mobility, New York, Springer: 235-256.

INGLEBY, David (2012), "Ethnicity, migration and the "social determinants of health" Agenda", Psychosocial Intervention, 21, 3: 331-341.

INGLEBY, David (2011), “Good practice in health service provision for migrants”, En Bernd Rechel, Philipa Mladovsky, Walter Devillé, Barbara Rijks, Roumyana Petrova-Benedict \& Martin McKee (Eds.), Migration and health in the European Union, European Observatory on Health Systems and Policies Series. Berkshire: McGraw Hill - Open University Press: 227-242.

INGLEBY, David; CHIARENZA, Antonio; DEVILLÉ, Walter \& KOTSIONI, Ioanna (Eds.) (2012), Inequalities in Health Care for Migrants and Ethnic Minorities, Vol. II, Antwerp/Apeldoorn: Garant.

INGLEBY, David; KRASNIK, Allan; LORANT, Vincent, \& RAZUM, Oliver (Eds.). (2012), Health inequalities and risk factors among migrants and ethnic minorities, Vol. I, Antwerp/Apeldoorn, Garant.

IOE (2014), "Las políticas neoliberales profundizan la desigualdad social”, Papeles de relaciones ecosociales y cambio global, 126: 57-69.

IOE (1998), “Inmigración y diversidad social en la España de fi n de siglo", Documentación Social, 111: 233-248.

KLEINMAN, Arthur \& BENSON, Peter (2006), “Anthropology in the clinic: the problem of cultural competency and how to fi x it” PLoS Medicine, 3, 10, e294, doi:10.1371/journal.pmed.0030294.

LEININGER, Madeleine (1978), Transcultural Nursing, New York, Wiley.

LEWIS, Gwyneth (Ed) (2007), The Confidential enquiry into maternal and child health (CEMACH). Saving mothers' lives: reviewing maternal deaths to make motherhood safer 2003-2005, The Seventh report on confidential enquiries into maternal deaths in the United Kingdom, London, CEMACH.

LUQUE, Miguel Ángel; BUENO, Aurora \& DE MATEO, Salvador (2010), “Differences in the reproductive pattern and low birthweight by maternal country of origin in Spain, 1996-2006", European Journal of Public Health, 21, 1: 104-108.

LUQUE, Miguel Ángel; GUTIÉRREZ, Ignacio \& BUENO, Aurora (2010), “Increased risk of maternal deaths associated with foreign origin in Spain: a population based case-control study", European Journal of Public Health, 21, 3: 292-294.

MACHADO, María do Céu; SANTANA, Paula; CARREIRO, Helena; NOGUEIRA, Helena; BARROSO, Rosalina, \& DIAS, Alexandra (2007), "Cuidados de saúde materna e infantil a uma população de imigrantes”, Migrações, 1: 103-127. 
MASANET, Erika; PADILLA, Beatriz; ORTIZ, Alejandra; HERNÁNDEZ-PLAZA, Sonia \& RODRIGUES, Elsa (2014), “Barreiras Socioeconómicas no acesso à Saúde Materno-Infantil das Mulheres Imigrantes na Área Metropolitana de Lisboa”, En Maria Inês Carsalade Martins, Ana Paula Marques, Nilson do Rosário Costa \& Alice Matos (Orgs.), Trabalho em Saúde, Desigualdades e Políticas Públicas, Braga, CICS: 17-29.

MFCCH (2012), Project to develop standards for equity in health care for migrants and other vulnerable groups. Preliminary standards for pilot testing in health care organizations, HPHWHO Task Force on Migrant-Friendly and Culturally Competent Healthcare, Reggio Emilia, Italia.

MINSART, Anne; ENGLERT, Yvon, \& BUEKENS, Pierre (2012), "Naturalization of immigrants and perinatal mortality”, European Journal of Public Health, 23, 2: 269-274.

MONTERO, Maritza (2006), Hacer para transformar. El método en la psicología comunitaria, Buenos Aires, Paidós.

NAVARRO, Vicenç (2014a), "El porqué del escandaloso crecimiento de las desigualdades sociales", Columna: Pensamiento crítico, Diario Público, 21 de octubre de 2014.

NAVARRO, Vicenç (2014b), “El desastre de las políticas de austeridad”, Columna: Dominio Público, Diario Público, 23 de octubre de 2014.

NAVARRO, Vicenç (2012), “The crisis and fi scal policies in the peripheral countries of the Eurozone", International Journal of Health Services, 42, 1: 1-7.

OMH (2001), National standards for culturally and linguistically appropriate services in health care. Final report, U.S. Department of Health and Human Services, Rockville, Offi ce of Minority Health.

ONDR (2013), Relatório do Observatório Nacional das Doenças Respiratórias 2013. Prevenir a doença, acompanhar e reabilitar o doente, Lisboa, ONDR.

OPSS (2014), Saúde Síndroma de negação. Relatório de Primavera 2014, Lisboa, Observatório Português dos Sistemas de Saúde.

PADILLA, Beatriz (2013), "Saúde dos imigrantes: multidimensionalidade, desigualdades e acessibilidade em Portugal", Revista REMHU, 21(40): 49-68.

PADILLA, Beatriz; HERNÁNDEZ PLAZA, Sonia; DE FREITAS, Cláudia; MASANET, Erika; SANTINHO, Cristina \& ORTIZ, Alejandra (2013), “Cidadania e diversidade em saúde: Necessidades e estratégias de promoção de equidade nos cuidados”, Saúde \& Tecnologia, Julio: 57-64.

PADILLA, Beatriz \& PEREIRA MIGUEL, José (2009), "Health and migration in the European Union: Building a shared vision for action", en Ana Fernandes \& José Pereira Miguel (Eds), Health and migration in the European Union: Better health for all in an inclusive society, Lisboa: Instituto Nacional de Saúde Doutor Ricardo Jorge: 101-115.

PADILLA, Beatriz; PORTUGAL, Rui; INGLEBY, David; DE FREITAS, Cláudia \& LEBAS, Jacques (2009), "Health and migration in the European Union: Good practices", En Ana Fernandes \& José Pereira Miguel (Eds.), Health and migration in the European Union: Better health for all in an inclusive society, Lisboa: Instituto Nacional de Saúde Doutor Ricardo Jorge: 101-115.

PHILLIMORE, Jenny (2011), "Approaches to health provision in the age of super-diversity: Accessing the NHS in Britain's most diverse city", Critical Social Policy, 31: 5-29.

RENSCHLER, Isabelle \& CATTACIN, Sandro (2007), “Comprehensive difference sensitivity in health systems", En Carin Bjömgren-Cuadra \& Sandro Cattacin (Eds.) Migration and health: Difference sensitivity from an organizatioal perspective: 37-41, Malmö, IMER. 
SEF (2012), Relatório de imigração, fronteiras e asilo 2011, Oeiras, Serviço de Estrangeiros e Fronteiras.

SEKN (2008), Understanding and tackling social exclusion. Final report to the WHO Commission on Social Determinants of Health from the Social Exclusion Knowledge Network. Geneva, World Health Organization.

SUAREZ BALCAZAR, Yolanda \& RODAKOWSKY, Juleen (2007), "Becoming a culturally competent occupational therapy practitioner", OT Practice, 24: 14-17.

SUE, Stanley; ZANE, Nolan; NAGAYAMA HALL, Gordon \& BERGER, Lauren (2009), "The case for cultural competency in psychotherapeutic interventions" Annual Review of Psychology, 60: 525-548.

TASHAKKORI, Abbas \& TEDDLIE, Charles (Eds.) (2003), Handbook of mixed methods in social and behavioural research, Thousand Oaks, Sage.

THOMAS, Felicity \& GIDEON, Jasmine (2013), Migration, Health and Inequality, London, Zed Books. VERTOVEC, Steven (2007), 'Super-diversity and its implications', Ethnic and Racial Studies, 29(6): 1024-1054.

WEAVER, Hillary (2008), "Striving for cultural competence: Moving beyond potential and transforming the helping professions", En Richard Dana \& James Allen (Eds.), Cultural competency training in a global society, New York, Springer: 135-155.

WHO Regional Office for Europe (2010), How health systems can address health inequalities linked to migration and ethnicity, Copenhagen, WHO Regional Office for Europe.

ZWART, Joost; JONKERS, Marina; RITCHERS, Annemiek; ORY, Ferko; BLOEMENKAMP, Kitty; DUVEKOT, Johannes \& ROOSMALEN, Jos (2010), "Ethnic disparity in severe acute maternal morbidity: a nationawide cohort study in the Netherlands" European Journal of Public Health, 21, 2: 229-234.

\section{NOTAS}

1. Los resultados que se describen en el presente artículo forman parte de un proyecto de investigación más amplio: "Meeting the healthcare needs of culturally diverse populations: A psycho-sociopolitical approach to cultural competence in health professionals" (PsySPOCUC), financiado en el marco del programa Marie Curie Actions FP7-PEOPLE-2010-IEF de la Comisión Europea (PIEF-GA-2010-272976) con la autora del presente artículo como investigadora principal. 2. "Meeting the healthcare needs of culturally diverse populations: A psycho-sociopolitical approach to cultural competence in health professionals" (PIEF-GA-2010-272976), Marie Curie Actions, Comisión Europea.

3. Los recortes en sanidad impuestos por la Troika se detallan en el documento: Portugal: Memorandum of Understanding on Specific Economic Policy Conditionality, 3 May 2011.

4. El Servicio Nacional de Salud (SNS) se crea en el año 1979 (Ley 56), fundamentado en los principios de universalidad, generalidad y gratuidad, como garantes del derecho a la salud recogido en la Constitución Nacional de 1976 (art. 64). En 1986 con la entrada de Portugal en la Comunidad Económica Europea, surge una preocupación por las condiciones para ejercer el derecho de acceso al SNS, abriéndose la posibilidad de introducir pagos directos por parte de los usuarios para acceder a los servicios sanitarios. En 1989, una enmienda a la Constitución elimina el atributo de "gratuidad"; y en 1992 se introduce el pago de "tasas moderadoras" por la utilización de los servicios sanitarios del SNS, incluyendo consultas médicas de atención primaria 
y especialidad, urgencias hospitalarias, pruebas complementarias y test diagnósticos, entre otros servicios (Hernández-Plaza, Padilla, Ortiz \& Rodrigues, 2014).

5. El aumento de las tasas moderadoras, en respuesta a las imposiciones de la Troika, se establece a través de la Portaria n. 306-A/2011 (Diário da República, 1. érie, n.․2 242, 20 de Diciembre de 2011).

6. La investigación-acción participativa es una modalidad de investigación social aplicada en la que los diferentes grupos implicados en el tema objeto de estudio participan de forma activa en el proyecto de investigación, no solamente como respondientes, diseñando de manera conjunta con los investigadores posibles acciones orientadas a cubrir las necesidades y resolver los problemas sociales objeto de análisis. La investigación-acción participativa basada en la comunidad se lleva a cabo a través del trabajo conjunto con organizaciones comunitarias y de la sociedad civil, implicadas activamente en los problemas objeto de investigación y acción.

7. Equipo interdisciplinar formado por las investigadoras: Beatriz Padilla, Sonia Hernández Plaza, Erika Masanet, Cristina Santinho, Alejandra Ortiz y Elsa Rodrigues.

8. PALOP, Países africanos de lengua oficial portuguesa: Cabo Verde, Angola, Mozambique, Guinea Bissau, São Tomé e Príncipe.

9. De acuerdo con los criterios establecidos por la Organización Mundial de la Salud (OMS), se considera edad reproductiva adulta la comprendida entre los 18 y 49 años.

10. Siguiendo la definición clásica originalmente planteada por Jonathan Bradshaw (1972), se consideran necesidades percibidas o sentidas aquellas definidas, identificadas o experimentadas por los propios afectados por un determinado problema o necesidad social.

11. Para un análisis psico-sociopolítico y multi-método de las necesidades identificadas en el proyecto de investigación, véase: Hernández-Plaza, S., Padilla, B., Ortiz, A. \& Rodrigues, E. (2014) The value of grounded theory for disentangling inequalities in maternal-child healthcare in contexts of diversity: a psycho-sociopolitical approach. Psychosocial Intervention, 23, 125-133.

12. Los datos procedentes de la encuesta fueron analizados mediante el programa IBM SPSS Statistics version 20, empleando la prueba no paramétrica Kruskal-Wallis para examinar las diferencias entre grupos en función del país de origen.

13. Los resultados presentados se integran asimismo dentro del proyecto de investigación acciónparticipativa: "Salud y ciudadanía: Disparidades y necesidades interculturales en la atención sanitaria a madres inmigrantes", financiado por la Fundação para a Ciência e a Tecnologia, Ministerio de Educación y Ciencia, Portugal (PTDC/CS-SOC/113384/2009), con la autora del presente artículo como miembro del equipo investigador, coordinado por la Prof. Beatriz Padilla. Este proyecto da protagonismo a la metodología cualitativa, incorporando asimismo algunos datos cuantitativos, como los analizados en el presente artículo, entre otros.

\section{RESÚMENES}

En el presente trabajo, se realiza un análisis crítico de la competencia cultural como estrategia para reducir las desigualdades en salud en contextos de diversidad asociada a la inmigración. Tras un breve recorrido histórico sobre el surgimiento y evolución de este concepto, se plantea una severa crítica a las políticas y prácticas de promoción de la competencia cultural dada su incapacidad para transformar las condiciones generadoras de desigualdad en el acceso a la salud y los servicios sanitarios en contextos de diversidad, particularmente en tiempos de crisis y austeridad. 
Neste trabalho, apresenta-se uma análise crítica da competência cultural como estratégia para reduzir as desigualdades em saúde em contextos de diversidade associada à imigração. Após um breve percurso histórico pelo surgimento e evolução deste conceito, faz-se uma severa crítica das políticas e práticas de promoção da competência cultural, dada a sua incapacidade para transformar as condições que levam à desigualdade na saúde e no acesso aos serviços de saúde em contextos de diversidade, particularmente nos atuais tempos de crise e austeridade.

The main aim of this paper is to critically examine the adequacy of cultural competence as a strategy to reduce health inequalities in contexts of migration-driven diversity. After a brief historical overview of the emergence and evolution of this concept, cultural competence policies and practices are severely criticized due to their inability to transform the conditions leading to inequalities in health and healthcare in contexts of diversity, particularly in current times of crisis and austerity.

\section{ÍNDICE}

Keywords: Cultural diversity, social inequalities, cultural competence, health, immigration, crisis, austerity

Palavras-chave: Diversidade cultural, desigualdades sociais, competência cultural, saúde, imigração, crises, austeridade

Palabras claves: Diversidad cultural, desigualdades sociales, competencia cultural, salud, inmigración, crisis, austeridade

\section{AUTOR}

\section{SONIA HERNÁNDEZ PLAZA}

Centro de Investigação em Ciências Sociais, Instituto de Ciências Sociais da Universidade do Minho. Endereço para correspondência: Centro de Investigação em Ciências Sociais (CICS), Universidade do Minho, Campus de Gualtar, 4710-057 Braga, Portugal sonia.h.plaza@gmail.com 